\section{Uso de PowerPoint en clases: ¿es necesario estudiarlo?}

Using PowerPoint in class: Is it necessary to study it?

\section{Señor Editor:}

En la actualidad, la mayoría de clases dictadas se realizan en PowerPoint, siendo esta una herramienta útil y de fácil acceso. Sin embargo, el uso inadecuado del PowerPoint puede afectar la efectividad en la enseñanza ${ }^{1,2}$. Existen recomendaciones acerca de tipo de fuente, palabras por viñeta y número de diapositivas usadas, que resultan útiles en general para orientar a un uso adecuado de las diapositivas en relación a la densidad de información ${ }^{3}$, incluso el uso de multimedia se ha encontrado efectividad en la enseñanza ${ }^{4}$. Por ello analizamos estas características en las diapositivas de las clases dictadas para el primer año de la carrera profesional de medicina humana en la facultad de Medicina de la Universidad Nacional Mayor de San Marcos en el año 2012.

Se analizaron el tipo de fuente, el número de palabras por viñeta y la cantidad de diapositivas de 138 clases de 8 cursos; las características fueron medidas mediante el mismo programa PowerPoint. El número de tipos de fuente varió entre de 1 a 18 para cada clase, $89 \%$ tuvo 
más de dos tipos de fuente en cada presentación, 57\% tuvo más de 6 palabras por viñeta y $38 \%$ tuvo más de 60 diapositivas.

La mayoría de las clases tuvo más de dos tipos de fuente y más de 6 palabras por viñeta. Es recomendable que el número de tipos de fuente debe ser menor de 2 y que el número de palabras por viñeta debe ser 6 para no cansar al auditorio y no desviar la atención del expositor hacia las diapositivas. Por otro lado, el número de diapositivas debe de ser lo adecuado como para poder ser explicadas en una clase, en nuestro caso $37 \%$ tenía más de 60 diapositivas, considerando una clase con una duración máxima de 2 h tendríamos un tiempo menor de dos minutos por diapositiva por lo que se corre el riesgo de dar mucha información sin que se llegue a entender ${ }^{1,3}$.

Si bien es cierto que estas sugerencias para la realización de PowerPoint nos pueden orientar a un uso adecuado, éstas no pueden seguirse rigurosamente debido a que cada clase debe de adaptar el uso de PowerPoint al tipo de información y al objetivo del profesor para cada clase, por lo que resulta complicado analizar la calidad y efectividad de una clase a partir de solo la descripción de las diapositivas en PowerPoint ${ }^{2,5,6}$.

Es así que resulta importante recalcar que esto es solo una descripción de las diapositivas, donde no podemos inferir sobre la calidad ni efectividad de la clase, pero si es posible decir que existen clases con alta cantidad de información que posiblemente no puedan ser explicadas en su totalidad ${ }^{1,2}$. Consideramos que a pesar de las limitaciones, estos resultados nos sugieren analizar la efectividad del uso de PowerPoint en las clases y no pasar por alto este problema que aún no es muy investigado en nuestro medio, y de esta manera poder encontrar la mejor forma de transmitir la información en una clase.

Paul Jesús Tejada-Llacsa ${ }^{1,2}$, Judith Cahuana-Aparco ${ }^{1,2,}$ ${ }^{1}$ Universidad Nacional Mayor de San Marcos. Lima, Perú.

${ }^{2}$ Asociación para el Desarrollo de la Investigación Estudiantil en Ciencias de la Salud (ADIECS-UNMSM). Lima, Perú.

\section{Referencias}

1. López D. Reflexiones sobre el uso de transparencias en clase. Actas las XX JENUI Oviedo 2014; 11-8.

2. Savoy A, Proctor RW, Salvendy G. Information retention from PowerPoint ${ }^{\mathrm{TM}}$ and traditional lectures. Comput Educ. Elsevier Ltd; 2009; 52 (4): 858-67.

3. Holzl J. Twelve tips for effective PowerPoint presentations for the technologically challenged. Med Teach 1997; 19 (3): 175-9.

4. Pate A, Posey S. Effects of applying multimedia design principles in PowerPoint lecture redesign. Curr Pharm Teach Learn. Elsevier 2016; 1-5.

5. Apperson JM, Laws EL, Scepansky J A. An assessment of student preferences for PowerPoint presentation structure in undergraduate courses. Comput Educ 2008; 50 (1): 148-53.

6. Penciner R. Does PowerPoint enhance learning? Can J Emerg Med 2013; 15 (2): 109-12.

Correspondencia a:

Paul Jesus Tejada-Llacsa

Av. Gonzales Prada 719, Comas, Lima-Perú.

paultejada24@gmail.com 